\title{
A Biomechanical Model of the Human Tongue and Its Clinical Implications
}

\author{
Yohan Payan ${ }^{1}$, Georges Bettega ${ }^{1,2}$, and Bernard Raphaël ${ }^{2}$ \\ 1 Laboratoire TIMC/IMAG - UMR CNRS 5525 - Faculté de Médecine \\ Domaine de la Merci, La Tronche - France - Yohan.Payan imag.fr \\ 2 Service de Chirurgie Plastique et Maxillo-Faciale \\ Centre Hospitalier Universitaire de Grenoble - France
}

\begin{abstract}
Many surgical technics act on the upper airway in general, and on the tongue in particular. For example, tongue is one of the anatomical structures involved in the case of Pierre Robin syndrome, mandibular prognathism, or sleep apnoea syndrome.

This paper presents the biomechanical and dynamical model of the human tongue we have developed, and the method we have used to fit this model to the anatomical and physical properties of a given patient's tongue. Each step of the modeling process is precisely described: the soft tissues modeling through the Finite Element Method (geometrical design of the FE structure within the upper airway and representation of lingual musculature), and the motor control of the model with the corresponding dynamical simulations. Finally, the syndromes listed above are presented, with some focus on the clinical implications of the model.
\end{abstract}

\section{INTRODUCTION}

Tongue is one of the anatomical structures implicated in the collapse of the upper airway, in obstructive sleep apnoea ([13]). Its muscular structure is also affected in the neonate troubles known as the syndrome of Pierre Robin ([15], [17]), or in the mandibular prognathism ([1], [4]). The development of a biomechanical model of tongue structure seems thus interesting, to understand the role played by human tongue tissues in the case of those syndromes. It may also be useful to evaluate and simulate some of the implications of surgical acts. This paper will present the biomechanical model of the human tongue, originally developed in the framework of an articulatory speech synthetiser ([12]). We will insist here on the way we have fit this model to the physical properties of a given patient. Finally, some syndromes involving tongue structure will be presented, and the usefulness of our model, in relation to those syndromes, will be discussed.

\section{TONGUE ANATOMY}

Tongue arrangement consists of a complex interweaving of active structures, the muscular fibers, and passive ones, mainly formed by glands and mucosa. The fan- 
tastic dexterity of this articulator is due to its structure, which contains a large amount of muscles, each of them being highly innervated. Indeed, ten muscles or so are able to shape precisely tongue structure in its sagittal, transverse and coronal planes. One particularity of those muscles is that most of them are internal to the structure, and are then responsible for their own deformation. Among all muscles which act on tongue structure, seven have an important influence on its shaping: the genioglossus, the styloglossus, the hyoglossus, the verticalis, the transverse, and the superior and inferior parts of the longitudinalis (figure 1).

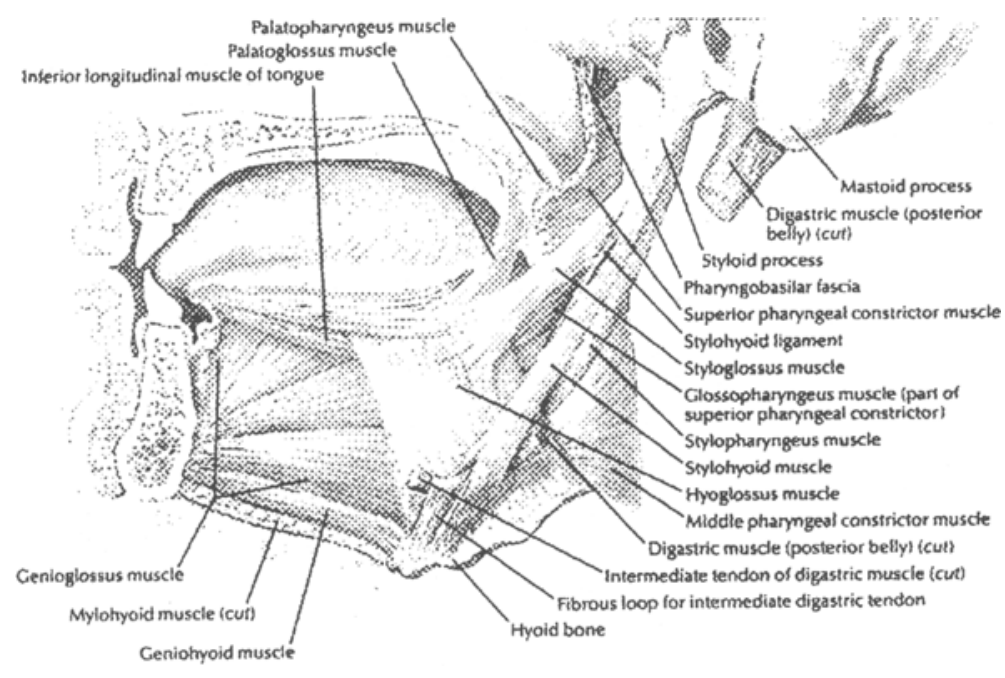

Fig. 1. Sagittal view of the human tongue musculature (from [11])

\section{THE TONGUE MODEL}

Perkell ([14]), with his 2D mass-spring model, has for the first time shown the interest of a physiological model of the tongue, to understand the respective contribution of each muscle in tongue's shaping. Following this concept, other biomechanical tongue models have been developed ([9], [18]). All of them have proposed a better analytical description of the continuous elastic structure of the tongue by the Finite Element Method. However, those models have not been evaluated and compared to measured tongue movements, as they were developed without any reference to the real measured properties of a given biological human tongue. To evaluate the ability of our model to precisely replicate the deformations observed on human tongue, we have decided to build a patient-specific model, i.e. the copy of the biological tongue of a human subject. 


\subsection{Definition of the model}

Our modeling approach was first dedicated to the choice of a mathematical tool able to describe the lingual deformations. Then, the structure was designed to fit the external shape of the reference patient's upper airways, and inserted into their corresponding contours. Finally, internal and external muscular fibers were defined, in order to give to the tongue structure its ability to move.

The Finite Element Method to model tongue soft tissues. The mathematical tool chosen to describe tongue deformation was the Finite Element Method (FEM). This method allows a precise description of the continuous, elastic and uncompressible properties of a body. For this, the physical properties of the body are taken into account through two elastic constants : the Young modulus $E$, which measures its stiffness, and the Poisson's ratio $\nu$, related to its compressibility. Moreover, it permits, via the notion of element, to attribute specific biomechanical properties to individual regions of the structure.

Geometrical shaping of the FE structure within the upper airways. Our aim was to develop a biomechanical model as close as possible to the morphological and physical characteristics of a given patient. That is the reason why the patient PB, who had already been subject to a large amount of articulatory data recordings (X-Ray, electropalatographies, electromagnetic recordings, MRI), was retained as our reference patient. Tongue description was limited to the midsagittal plane, in accordance with the fact that the main articulatory data available in the literature describe tongue movements in this sagittal plane. The first stage of tongue shaping was then to collect a given X-ray picture of PB's tongue, associated to a rest position of his lingual articulator. The corresponding radiography gives, in the midsagittal plane, the upper airways contours (hard palate, velar regions, pharynx and larynx), the shape and position of the mandible, the lips, the hyoid bone, and the external contour of the tongue, as a reference for the rest shaping of our model (figure 2.a). An isoparametric mesh, composed of 48 elements, was then defined and distributed inside the tongue structure. The FE tongue model was finally inserted into PB's upper airways contours (figure 2.b). Tongue insertions on jaw and hyoid bone were modeled by imposing no displacements of the corresponding nodes.

Tongue muscles modeling. The great majority of tongue muscles are left/right muscle pairs, i.e. muscles composed of two symmetrical parts along the sagittal plane. Our modeling approach has considered the two symmetrical parts of each tongue muscle as a unique entity, acting in the sagittal plane. Furthermore, we have only modeled muscles whose action has significant influence on tongue shaping in the sagittal plane, namely the posterior and anterior parts of the genioglossus, the hyoglossus, the styloglossus, the inferior and superior longitudinalis, and the verticalis. 


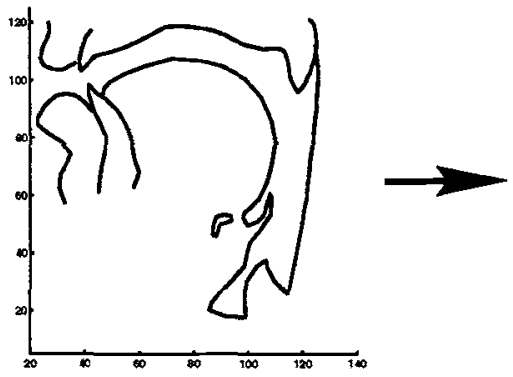

(a)

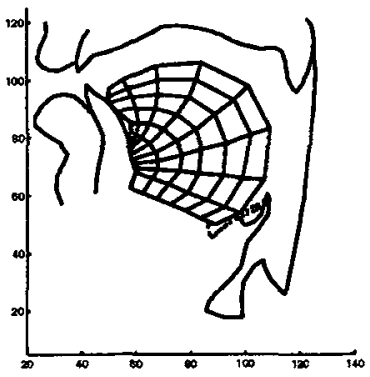

(b)

Fig. 2. The 48 elements (63 nodes) building the model are shaped (b) to give the model the rest configuration of the patient's tongue (a).

Choice for the FE elastic parameters. Young modulus $E$ and Poisson's ratio $\nu$ values have to be carefully chosen, as they directly influence the way the FE structure will be deformed under muscular actions. The choice for the $\nu$ value was the simplest one, because of the specific tongue physiological structure. Indeed, tongue tissues are mainly composed of water (see for example the density of $1040 \mathrm{~kg} / \mathrm{m}^{3}$ reported by Duck ([6]) for human muscle tissues). In order to model this quasi-incompressibility, a. $0.49 \nu$ value was chosen. As concerns the Young modulus, its value is directly related to the stiffness of tongue body. If no data, from our knowledge, are available in the literature about $E$ value for tongue tissues, some measurements are reported for other soft part of human body ([10], [6]). Moreover, some measurements of forces developed by each tongue muscle during speech production were provided by Bunton and Weismer ([2]). Those levels of forces were used to act on tongue muscles, in order to compare the FE deformations with the lingual deformations measured on PB during some speech sequences. This helped us in tuning the Young modulus value, which was finally fixed to $15 \mathrm{kPa}$. Once the elastic parameters have been chosen, the lingual deformations induced by each modeled muscle can be computed. Figure 3 plots the actions of four muscles, namely the posterior part of the genioglossus, the hyoglossus, the superior longitudinalis, and the styloglossus. Each muscle is composed of one to three macro-fibers, on which global muscle force is distributed.

Dynamics in the model. The biomechanical model of the tongue is now able to be animated under the coordination of all the modeled muscles. For this, we have retained, for the control of tongue movements, the concepts developped by feldman ([7]), and proposed more generally for the control of human limb movements. Those propositions are based on a functional model of force generation, for which the level of force depends on muscle length and rate of length from one hand, and on a central command, called the " $\lambda$ command", on the other hand (see [12] for more details about this neurophysiological model for force generation). Following this hypothesis, it is assumed that the Central Nervous System 


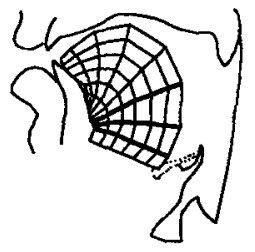

(a) Posterior Genioglossus

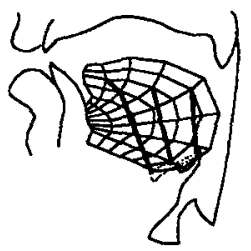

(b) Hyoglos-

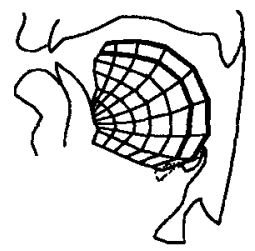

(c) Superior longitudinalis

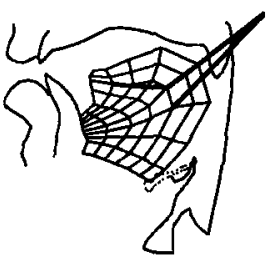

(d) Styloglossus

Fig. 3. Contribution of some tongue muscles to the global tongue shaping. The macrofibers, modeling the muscles forces, are shown by the bold lines.

controls the temporal evolution of each muscular $\lambda$ command. The corresponding movements of the FE tongue structure has then to be computed. For this, the temporal evolution of $U, U$ and $\ddot{U}$, respectively the displacement, velocity and acceleration vectors (dimension: $63 \times 2$ ) of the structure's nodes, is computed by solving the equation of motion via the adaptive Runge Kutta method :

$$
M \ddot{U}+f \dot{U}+K(U, \dot{U}, \lambda) U=F(U, \dot{U}, \lambda)+P
$$

$M$ is the global mass matrix ( $100 \mathrm{~g}$ for the whole tongue);

$f$ is the global passive damping matrix (viscosity coefficient $=15 \mathrm{~N} \mathrm{~s} / \mathrm{m}$ ) ;

$K$ is the stiffness matrix, modeling the passive elastic behaviour of the tongue and computed by means of the FE technique ; this matrix depends on central commands, muscle length and rate of muscle length change, since some elements have a Young modulus modified by muscle activation ;

$F$ is a vector of forces exerted by muscles, and concentrated on specific nodes ; $P$ is the vector of all external forces acting on each node : gravity and contact forces (tongue against the teeth or the palate).

Figure 4 shows the successive tongue shapes obtained with a linear shift of the hyoglossus and posterior genioglossus central commands (patterns used to move the tongue from a front and high position towards a back and low one).

\section{CLINICAL IMPLICATIONS OF THE MODEL}

Some implications could be drawn concerning the usefulness of a biomechanical modeling, at the clinical level. In particular, it is interesting to focus on syndromes involving tongue structure, namely the Pierre Robin syndrome ([15], [17]), mandibular prognathism ([1], [4]), or sleep apnoea syndrome ([13]).

\subsection{Pierre Robin Syndrome}

The combination of micro-retromandibulism, glossoptosis, palatal clefting and respiratory troubles observed in the neonate is known as the syndrome of Pierre 


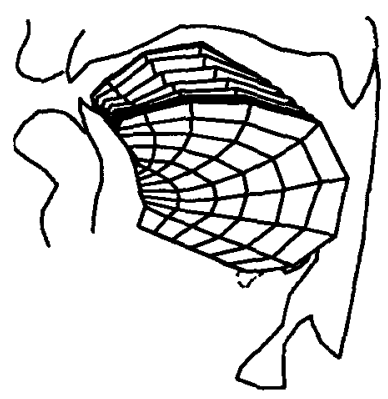

Fig. 4. Dynamical tongue deformations under the coordinated action of posterior genioglossus and hyoglossus muscles.

Robin ([15]). The pathogenesis is a disturbance of muscular maturation of nervous origin and the syndrome belongs to the category of muscular dysmaturations which affects the masticatory muscles, the tongue and the pharyngeal slings. Swallowing is disturbed and airway obstructed, resulting in the aspiration of secretion and foods. The respiratory difficulties are further increased by the low and posterior position of the tongue. A lateral radiographic soft tissue view shows the ptotic tongue to be positioned below the mandibular angle, pressing on the epiglottis. Clinical inspection shows three crests: mandibular, sublingual and lingual. The malformation is characterised by a functional disturbance of the tongue which may be associated with a palatal cleft. The degree of lingual protrusion is a criterion of the evolution of the syndrome. Various treatments are possible. Skilled nursing care with the child in prone position, maintained by a frame, is the treatment most commonly used ([3]). Application of a palatal plate to hold the tongue in forward position and lingual rehabilitation by stimulation of suction are complementary methods of treatment. Some cases are surgically treated, either by mandibulohyoid fixation or better by a submental myoplasty ([5]) ; the tracheotomy is exceptionally used.

The biomechanical model of the tongue could be useful to obtain a prognostic factor of the neuro-muscular dysmaturation. This would help to precise the therapeutic strategy and should give objective elements for the follow-up.

\subsection{Mandibular prognathism}

Dentofacial deformity of the lower third of the face resulting from excess mandibular growth is not as common as mandibular deficiency. However the frequency of class III malocclusion (anterior placement of mandibular teeth compared with maxillary teeth) is approximately 1 to $3 \%$ of the population, depending on age and geographic variation. The deformity is related to an excess of mandibular development or to a retrusion of maxilla, but the majority of patients associate both abnormalities. The etiology of mandibular excess has been studied extensively, but the primary determinant is still controversial. However, tongue posi- 
tion and tongue function seem to be an important factor in the etio-pathogeny of the problem, and seem to be one of the major causes of relapse of the dysmorphosis after treatment (either orthodontic or surgical). Very often, the tongue of those patients is flat and anteriorly displaced, this causes a widened mandibular arch and a narrow maxillary arch with high palate. The goal of treatment is to correct the occlusion (dental position) either with an isolated orthodontic work or with an association of orthodontics and orthognathic surgery. But the therapeutic plan must include the correction of tongue position and function to avoid relapse. Usually this part is assumed by the orthophonist, but in some cases surgical tongue reduction (glossectomy) should be discussed, even if controversial. In the framework of the mandibular prognathism, the implications of a biomechanical model of the human tongue are numerous :

- To identify and appreciate the participation of tongue malposition or of tongue dysfunction in the etiology of mandibular prognathism.

- To identify the patients who present a risk of relapse after treatment (orthodontic or surgical treatment) in a way to prevent this by activation of the re-education by the orthophonist.

- To prove the uselessness of glossectomy in the treatment of prognathism.

- The same protocol should be extended to the dysmorphosis with an open bite anomaly. In this case the problem seem to be, very often, the interposition of the tongue between dental arches.

\subsection{Sleep apnoea syndrome}

Airway closure during sleep apnoea syndrome (SAS) comes from the interaction between inspiratory flow and the upper airway that is formed by the succession of anatomical and physiological singularities with their own geometrical and mechanical properties ([13]). The tongue is one of the anatomical structures implicated in the collapse. Some of the surgical techniques associated to the SAS reduce the volume of the tongue, while others try to have a more global and progressive action on the entire upper airway. In order to fully understand the metamorphosis in inspiratory flow pattern that has been reported during SAS, some mechanical models of the upper airway have been developed, assuming that upper airways can be represented by a single compliant segment ([16]), or by series of individual segments representing singularities ([8]).

In this framework, a complete biomechanical model of the tongue appears thus to be interesting, to describe and explain, at the mechanical point of view, the upper airway obstruction.

\section{CONCLUSION}

The biomechanical model of the tongue we have developed was presented in details, with specific focuses on its relation to the physical properties of a given patient. Special emphasis was put onto the shaping of the FE tongue structure, 
the definition of muscular fibers, as well as the choice of FE elastic parameters. Some points supporting an anthropomorphic modeling and its use for clinical applications were given and discussed.

\section{ACKNOWLEDGEMENTS}

The biomechanical work was carried out with Pascal Perrier, from the ICP (Grenoble, France), and supported by the grant INSERM PARMPSTRC 9607.

\section{References}

1. H. Bell. Modern practice in orthognathic and reconstructive surgery, volume 3 . WB Saunders company, Philadelphia, 1992.

2. K. Bunton and G. Weismer. Evaluation of a reiterant force-impulse task in the tongue. Journal of Speech and Hearing Research, 37:1020-1031, 1994.

3. W.R Burston. Neonatal surgery, chapter Mandibular retrognathia. Rickham P.P. and Johnston J.H. eds, London, 1969.

4. J. Delaire. la langue et dysmorphie, chapter la langue et dysmorphie, page 81. Masson, Paris, 1996.

5. R.P Delorme, Y. Laroque, and L. Caouette-Laberge. Innovative surgical approach for the pierre robin anomalad : subperiostal release of the floor of the mouth musculature. Plast. Reconstr. Surg., 83:960-64, 1989.

6. F.A. Duck. Physical properties of tissues: a comprehensive reference book. Academic Press, London, 1990.

7. A.G. Feldman. Once more on the equilibrium-point hypothesis ( $\lambda$ model) for motor control. Journal of Motor Behavior, 18(1):17-54, 1986.

8. R. Fodil, C. Ribreau, B. Louis, F. Lofaso, and D. Isabey. Interaction between steady flow and individualised compliant segments: application to upper airways. Medical ES Biological Engineering 83 Computing, November 1997.

9. K. Honda. Organization of tongue articulation for vowels. Journal of Phonetics, 24:39-52, 1996.

10. Y. Min, I. Titze, and F. Alipour. Stress-strain response of the human vocal ligament. NCVS Status and Progress, 7:131-137, 1994.

11. F.H Netter. Atlas of human anatomy. Technical report, CIBA-GEIGY Corporation editor, 1989.

12. Y. Payan and P. Perrier. Synthesis of v-v sequences with a $2 \mathrm{~d}$ biomechanical tongue model controlled by the equilibrium point hypothesis. Speech Communication, 22(2-3):185-205, 1997.

13. J.L Pépin, D. Veale, and G. Ferreti. Evaluation of the upper airway in sleep apnea syndrome. Sleep, 15:s50-s55, 1992.

14. J.S Perkell. A physiologically-oriented model of tongue activity in speech production. $\mathrm{PhD}$ thesis, Massachusetts Institute of Technology, Boston, 1974.

15. P. Robin. La glossoptose. Un grave danger pour nos enfants. Doin ed., Paris, 1929.

16. P.L. Smith, R.A. Wise, A.R. Gold, A.R. Schwarts, and S. Permutt. Upper airway pressure-flow relationship in obstructive slepp apnea. J. Appl. physiol, 64:789-795, 1998.

17. M. Stricker, J. Van Der Meulen, B. Raphael, and R. Mazzola. Craniofacial malformations. Churchill Linvingstone ed., Edingburgh, 1990.

18. R. Wilhelms-Tricarico. Physiological modeling of speech production: Methods for modeling soft-tissues articulators. J. Acoust. Soc. of Am., 97(5):3085-3098, 1995. 